\title{
N, S, and P-co-doped Carbon Quantum Dots: Intrinsic Peroxidase Activity in a Wide pH Range and its Antibacterial Applications
}

Kumud Malika Tripathi ${ }^{a}$, Hee Tae Ahn ${ }^{\mathrm{b}}$, Minsoo Chung ${ }^{\mathrm{b}}$, Xuan Ai Le ${ }^{\mathrm{b}}$, Deepika Sainic ${ }^{\mathrm{c}}$, Anshu Bhatic, Sumit Kumar Sonkarc, Moon Il Kim ${ }^{\mathrm{b} *}$, TaeYoung Kim ${ }^{\mathrm{d} *}$

a Department of Chemistry, Indian Institute of Petroleum and Energy, Visakhapatnam 530003, Andhra Pradesh, India

${ }^{\mathrm{b}}$ Department of BioNano Technology, Gachon University, 1342 Seongnam-daero, Sujeong-gu, Seongnam, Gyeonggi 13120, South Korea

${ }^{c}$ Department of Chemistry, Malaviya National Institute of Technology, Jaipur 302017, India

${ }^{d}$ Department of Materials Science and Engineering, Gachon University, 1342 Seongnam-daero, Sujeong-gu, Seongnam, Gyeonggi 13120, South Korea

"Corresponding author(s) E-mail: moonil@gachon.ac.kr and taeykim@gachon.ac.kr $\square$ equal contribution 

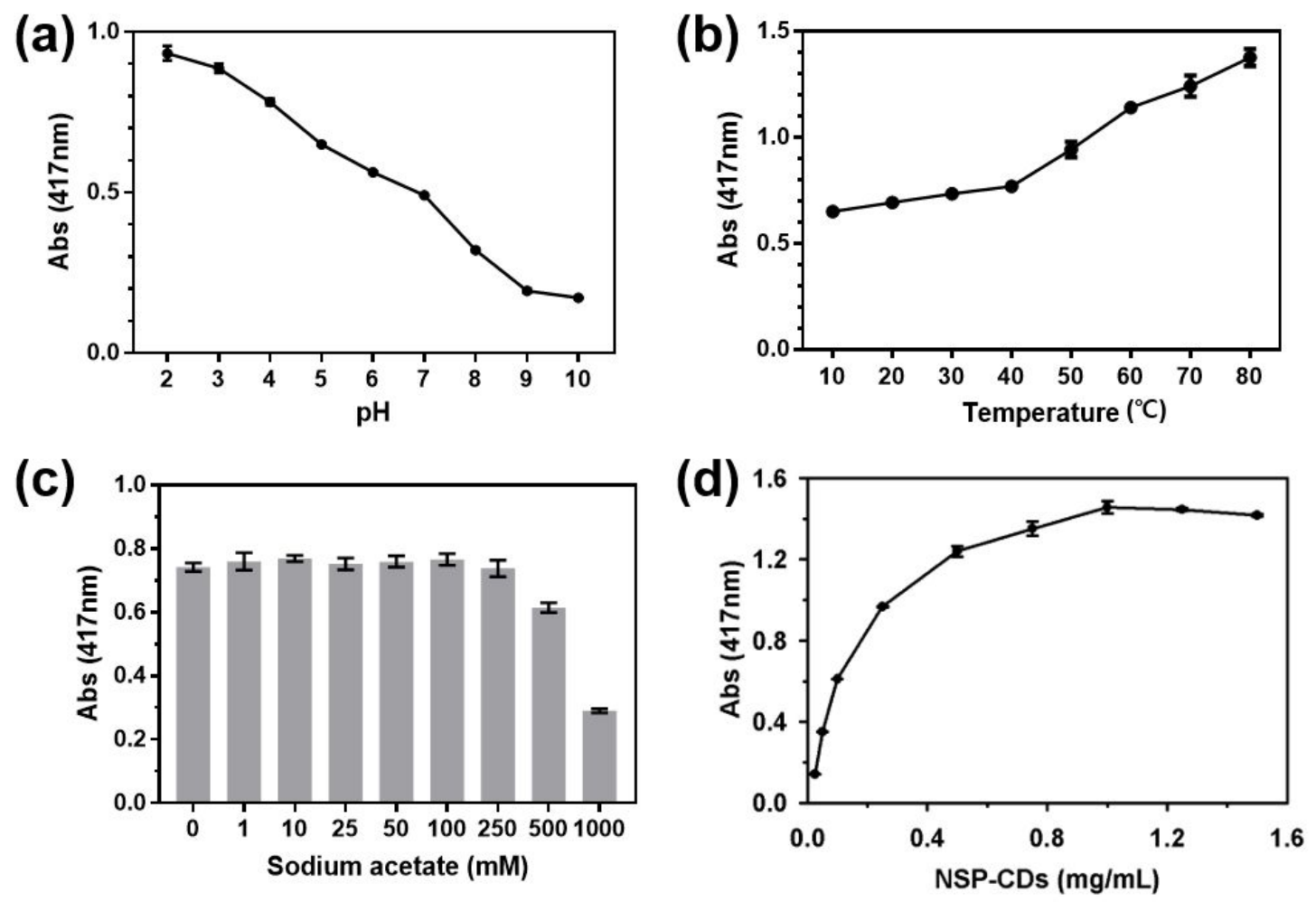

Figure S1. Effect of (a) $\mathrm{pH}$; (b) temperature, (c) ionic strength, and (d) concentration of NSPCQDs on the catalytic activity for ABTS oxidation by NSP-CQDs in co-existence of $\mathrm{H}_{2} \mathrm{O}_{2}$. 

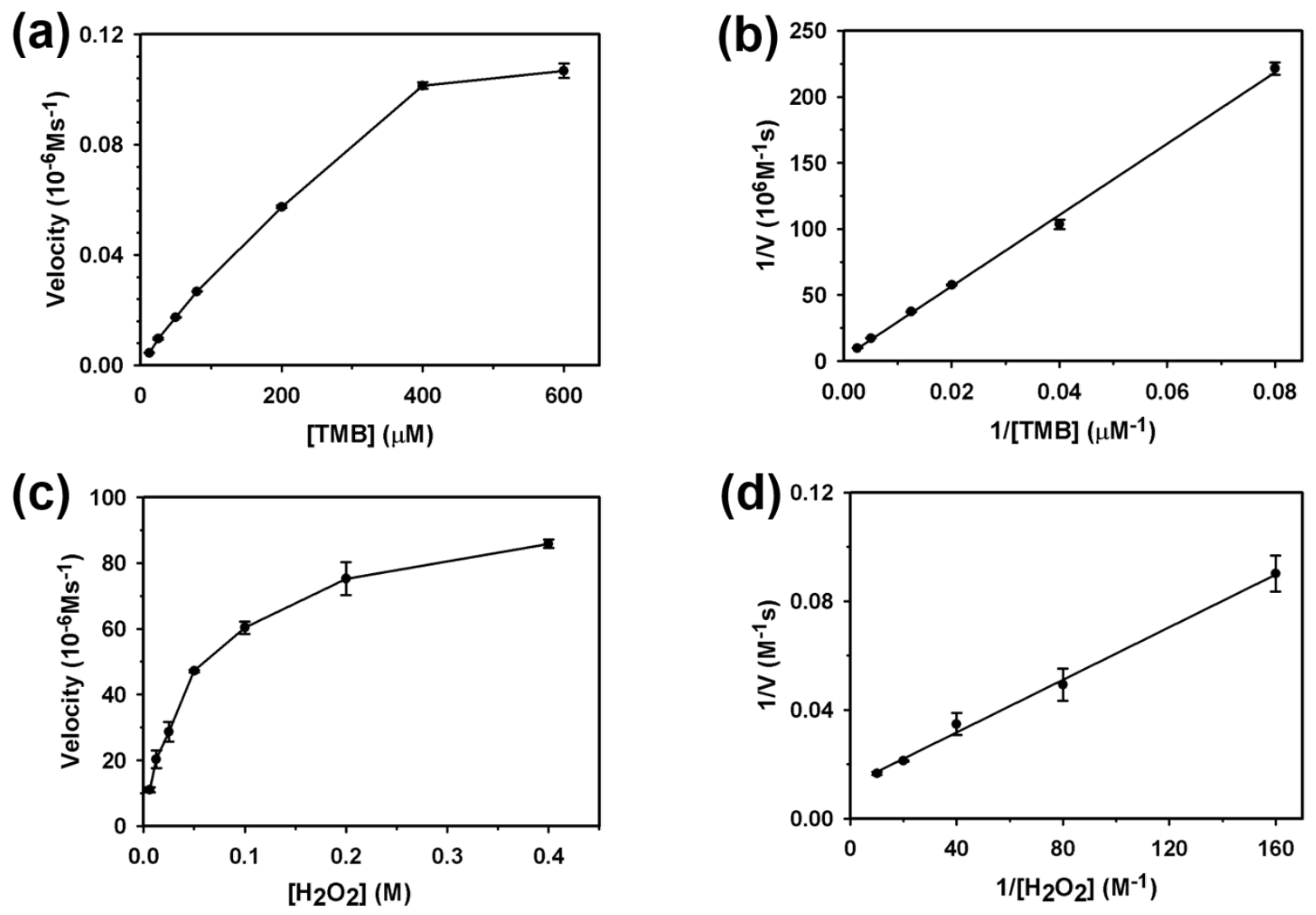

Figure S2. Steady-state kinetic assays of NSP-CQDs with diverse concentrations of (a, b) TMB and (c, d) $\mathrm{H}_{2} \mathrm{O}_{2}$. 

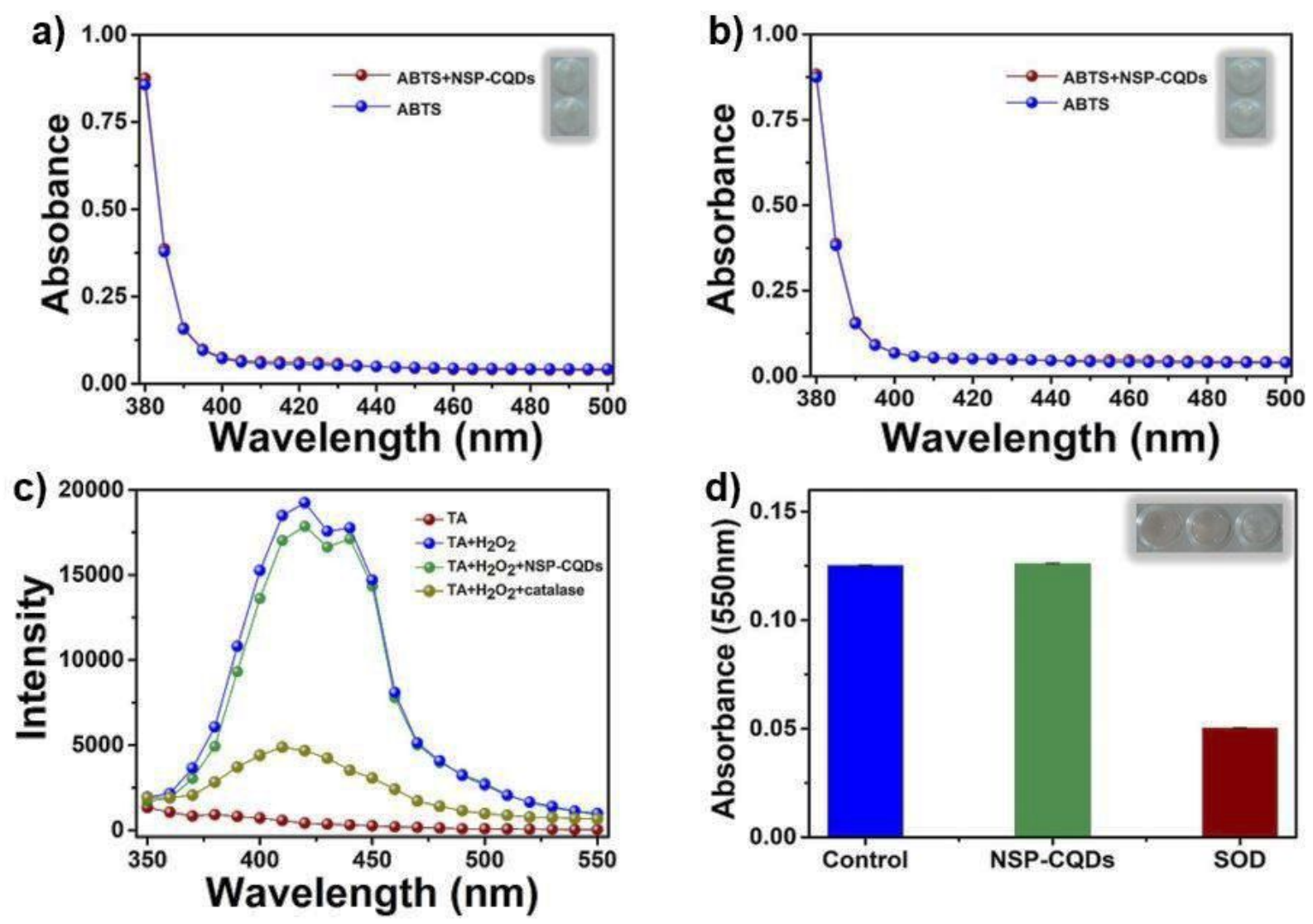

Figure S3. Evaluations of oxidase-, catalase-, and SOD-like activity of NSP-CQDs. Oxidaselike activity at (a) pH 4 and (b) $\mathrm{pH} 7$; (c) catalase-like activity and (d) SOD-like activity.

Oxidase-like activity of NSP-CQDs was determined using ABTS substrate in the absence of $\mathrm{H}_{2} \mathrm{O}_{2}$. In details, a solution containing $20 \mu \mathrm{L}$ of ABTS $(60 \mathrm{mM}), 20 \mu \mathrm{L}$ of NSP-CQDs $(500 \mu \mathrm{g} / \mathrm{mL})$ in $160 \mu \mathrm{L}$ of sodium acetate buffer $(50 \mathrm{mM}, \mathrm{pH} 4$ and $\mathrm{pH}$ 7) was incubated for $10 \mathrm{~min}$ at room temperature. After centrifugation, the supernatant was obtained to measure the corresponding absorbance to oxidized ABTS using a microplate reader (Synergy H1, BioTek, VT) in a scanning mode.

Catalase-like activity was measured using a solution containing terephthalic acid (TA, $0.5 \mathrm{mM})$, $\mathrm{H}_{2} \mathrm{O}_{2}(10 \mathrm{mM})$, and NSP-CQDs $(50 \mu \mathrm{g} / \mathrm{mL})$ in sodium acetate buffer $(50 \mathrm{mM}, \mathrm{pH}$ 7). Free catalase was used as the control. After $20 \mathrm{~min}$ exposure under UV light at $365 \mathrm{~nm}$ followed by 
centrifugation, the solution was used for fluorometric measurements with excitation wavelength of $315 \mathrm{~nm}$ using a microplate reader (Synergy H1, BioTek, VT).

SOD-like activity was measured using a solution containing xanthine $(100 \mu \mathrm{M})$, xanthine oxidase (5 mU/mL), catalyst samples (NSP-CQDs or free SOD enzyme, $50 \mu \mathrm{g} / \mathrm{mL})$, and cytochrome c (10 $\mu \mathrm{M})$ in sodium phosphate buffer (50 mM, pH 7.4). Xanthine oxidase catalyzed the oxidation of xanthine to produce $\mathrm{H}_{2} \mathrm{O}_{2}$, urate, as well as superoxide radicals $\left(\mathrm{O}_{2}{ }^{-}\right)$. After centrifugation for separation, the solutions were used for absorbance measurements at $550 \mathrm{~nm}$ using a microplate reader (Synergy H1, BioTek, VT).

Table S1. Comparison of the Michaelis constant $\left(\mathrm{K}_{\mathrm{m}}\right)$ and maximal reaction velocity $\left(\mathrm{V}_{\max }\right)$ of NSP-CQDs with those from other peroxidase-mimicking nanozymes and HRP.

\begin{tabular}{|c|c|c|c|c|}
\hline & Substrate & $K_{m}[m M]$ & $\mathrm{V}_{\max }\left(10^{-8} \mathrm{Ms}^{-1}\right)$ & References \\
\hline \multirow[t]{2}{*}{ HRP } & TMB & 2.39 & 4.36 & \multirow{2}{*}{1} \\
\hline & $\mathrm{H}_{2} \mathrm{O}_{2}$ & 0.18 & 0.29 & \\
\hline \multirow[t]{2}{*}{ HRP } & TMB & 5.9 & 183.33 & \multirow{2}{*}{2} \\
\hline & $\mathrm{H}_{2} \mathrm{O}_{2}$ & 0.63 & 2250.00 & \\
\hline \multirow[t]{2}{*}{ HRP } & TMB & 0.434 & 10.00 & \multirow{2}{*}{3} \\
\hline & $\mathrm{H}_{2} \mathrm{O}_{2}$ & 3.702 & 8.71 & \\
\hline \multirow[t]{2}{*}{ CDs } & TMB & 0.039 & 3.61 & \multirow{2}{*}{4} \\
\hline & $\mathrm{H}_{2} \mathrm{O}_{2}$ & 26.77 & 30.61 & \\
\hline \multirow[t]{2}{*}{ BNS-CDs } & TMB & 0.44 & 9.47 & \multirow{2}{*}{5} \\
\hline & $\mathrm{H}_{2} \mathrm{O}_{2}$ & 0.023 & 1.26 & \\
\hline \multirow[t]{2}{*}{ Co-CDs } & TMB & 0.32 & 5.45 & \multirow{2}{*}{6} \\
\hline & $\mathrm{H}_{2} \mathrm{O}_{2}$ & 4.81 & 4.68 & \\
\hline \multirow[t]{2}{*}{ Pd-CDs } & TMB & 0.74 & 7666.67 & \multirow{2}{*}{7} \\
\hline & $\mathrm{H}_{2} \mathrm{O}_{2}$ & 10.12 & 1200.00 & \\
\hline \multirow[t]{2}{*}{$\mathrm{N} \& \mathrm{Cu}-\mathrm{CDs}$} & TMB & 1.76 & 0.30 & \multirow{2}{*}{8} \\
\hline & $\mathrm{H}_{2} \mathrm{O}_{2}$ & 11.91 & 0.55 & \\
\hline
\end{tabular}




\begin{tabular}{ccccc}
\hline $\mathrm{V}_{2} \mathrm{O}_{5}-\mathrm{CDs}$ & $\mathrm{TMB}$ & - & - & 9 \\
& $\mathrm{H}_{2} \mathrm{O}_{2}$ & 53.6 & 35.1 & \\
$\mathrm{Fe}_{3} \mathrm{O}_{4} \mathrm{NPs}$ & $\mathrm{TMB}$ & 0.374 & 2.6 & 10 \\
& $\mathrm{H}_{2} \mathrm{O}_{2}$ & 54.6 & 1.8 & \\
\multirow{2}{*}{$\mathrm{NSP}-\mathrm{CQDs}$} & $\mathrm{TMB}$ & 0.47 & 18.81 & This work \\
& $\mathrm{H}_{2} \mathrm{O}_{2}$ & 32.61 & 6950.68 & \\
\hline
\end{tabular}

\section{References:}

1. Lin, L.; Song, X.; Chen, Y.; Rong, M.; Zhao, T.; Wang, Y.; Jiang, Y.; Chen, X., Intrinsic peroxidase-like catalytic activity of nitrogen-doped graphene quantum dots and their application in the colorimetric detection of $\mathrm{H}_{2} \mathrm{O}_{2}$ and glucose. Anal. Chim. Acta, 2015, 869, 89-95.

2. Lu, C.; Liu, X.; Li, Y.; Yu, F.; Tang, L.; Hu, Y.; Ying, Y., Multifunctional janus hematite-silica nanoparticles: mimicking peroxidase-like activity and sensitive colorimetric detection of glucose. ACS Appl. Mater. Interfaces, 2015, 7, 15395-15402.

3. Jiao, X.; Song, H.; Zhao, H.; Bai, W.; Zhang, L.; Lv, Y., Well-redispersed ceria nanoparticles: promising peroxidase mimetics for $\mathrm{H}_{2} \mathrm{O}_{2}$ and glucose detection. Anal. Methods, 2012, 4, 32613267.

4. Shi, W.; Wang, Q.; Long, Y.; Cheng, Z.; Chen, S.; Zheng, H.; Huang, Y., Carbon nanodots as peroxidase mimetics and their applications to glucose detection. Chem. Commun., 2011, 47, 66956697.

5. Peng, B.; Xu, J.; Fan, M.; Guo, Y.; Ma, Y.; Zhou, M.; Fang, Y., Smartphone colorimetric determination of hydrogen peroxide in real samples based on B, N, and $\mathrm{S}$ co-doped carbon dots probe. Anal. Bioanal. Chem., 2020, 412, 861-870.

6. Guo, Y.; Liu, X.; Yang, C.; Wang, X.; Wang, D.; Iqbal, A.; Liu, W.; Qin, W., Synthesis and peroxidase-like activity of cobalt@ carbon-dots hybrid material. ChemCatChem, 2015, 7, 24672474. 
7. Zhuo, S.; Fang, J.; Zhu, C.; Du, J. J. A.; Chemistry, B., Preparation of palladium/carbon dot composites as efficient peroxidase mimics for $\mathrm{H}_{2} \mathrm{O}_{2}$ and glucose assay. Anal. Bioanal. Chem., 2020, 412, 963-972.

8. Lin, L.; Xiao, Y.; Wang, Y.; Zeng, Y.; Lin, Z.; Chen, X., Hydrothermal synthesis of nitrogen and copper co-doped carbon dots with intrinsic peroxidase-like activity for colorimetric discrimination of phenylenediamine isomers. Microchim. Acta, 2019, 186, 288.

9. Honarasa, F.; Kamshoori, F. H.; Fathi, S.; Motamedifar, Z. Carbon dots on $\mathrm{V}_{2} \mathrm{O}_{5}$ nanowires are a viable peroxidase mimic for colorimetric determination of hydrogen peroxide and glucose. Microchim. Acta, 2019, 186, 234.

10. Vallabani, N. S.; Karakoti, A. S.; Singh, S. ATP-mediated intrinsic peroxidase-like activity of $\mathrm{Fe}_{3} \mathrm{O}_{4}$-based nanozyme: one step detection of blood glucose at physiological pH. Colloid Surf. BBiointerfaces, 2017, 153, 52-60. 